\title{
Efecto de la Microencapsulación sobre la Viabilidad de Lactobacillus delbrueckii sometido a Jugos Gástricos Simulados
}

\author{
Rafael E. González Cuello(1), Jaime Pérez Mendoza(1), Lena B. Morón Alcázar ${ }^{(1)}$ \\ (1) Universidad de Cartagena, Piedra de Bolívar - Av Del Consulado, Calle 30 № 48-157., Colombia. \\ (e-mail: rgonzalezc1@unicartagena.edu.co)
}

Recibido Mar. 18, 2015; Aceptado May. 8, 2015; Versión final Jun. 4, 2015, Publicado Oct. 2015

\begin{abstract}
Resumen
En el presente trabajo se evaluó el efecto de un sistema microencapsulante binario compuesto por alginato de sodio (AS) y gelana de alto acilo (GAA) en la protección de Lactobacillus delbrueckii sometido a condiciones gástricas simuladas. La microencapsulación es una técnica que consiste en envolver un principio activo con el fin de protegerlo frente a condiciones ambientales deletéreas. Los resultados indicaron que es posible mantener la viabilidad de Lactobacillus delbrueckii en un $50 \%$ después de $4 \mathrm{~h}$ de exposición a jugos gástricos simulados cuando se utiliza AS Y GAA como sistema pared. Los microorganismos en estado libre pierden completamente su viabilidad a los 180 minutos de exposición. Por tal motivo, la microencapsulación podría ser una técnica útil en la producción de alimentos funcionales manteniendo los niveles terapéuticos de las bacterias probióticas.
\end{abstract}

Palabras clave: jugos gástricos simulados; microencapsulación; Lactobacillus delbrueckii; viabilidad

\section{Effect of Microencapsulation on the Viability of Lactobacillus delbrueckii under Simulated Gastric Juice}

\begin{abstract}
In this paper the effect of a microencapsulant binary system composed by sodium alginate (SA) and high acyl gellan (HAG) in the protection of Lactobacillus delbrueckii under simulated gastric conditions was evaluated. Microencapsulation is a technique that involves wrapping an active ingredient in order to protect them against deleterious environmental conditions. The results indicated that it is possible to maintain the viability of Lactobacillus delbrueckii at $50 \%$ after $4 \mathrm{~h}$ exposure to simulated gastric juices when AS and HAG are used as wall system. The free microorganisms completely lose their viability at 180 minute exposure. Therefore, microencapsulation can be a useful technique in the production of functional foods maintaining therapeutic levels of probiotic bacteria.
\end{abstract}

Keywords: simulated gastric juice; microencapsulation; Lactobacillus delbrueckii; viability 


\section{INTRODUCCIÓN}

Las bacterias probióticas son microorganismos vivos que al situarse en el tracto gastrointestinal pueden ejercer efectos benéficos en la salud del huésped. Algunos de estos efectos incluyen: control del colesterol, regulación de infecciones intestinales, mejora del sistema inmunológico, utilización de lactosa y disminución de actividad anticancerígena (Zhu et al., 2011; Tripathi y Giri, 2014). Cada una de estas propiedades dependen de la habilidad que presentan los probióticos de multiplicarse al interior del huésped, ya que para conferir los efectos benéficos mencionados previamente deben primero sobrevivir su paso a través del tracto gastrointestinal (Mohammadi et al., 2011). Dicha sobrevivencia se ve afectada por diversos factores: como la presencia o ausencia de oxígeno, el medio ambiente ácido, la interacción entre especies, las condiciones del cultivo, la producción de peróxido de hidrógeno por metabolismo microbiano, la disponibilidad de nutrientes y la presencia de inhibidores de crecimiento. Dentro de las bacterias probióticas, el género Lactobacillus spp ha sido uno de los más estudiados y utilizados en la producción de alimentos funcionales en los años recientes (Ribeiro et al., 2014). Por consiguiente, una estrategia que permita la protección de bacterias probióticas en especial del género Lactobacillus spp frente a condiciones adversas resulta llamativa para una posterior aplicación en múltiples sistemas alimentarios. Una técnica usualmente utilizada en la protección de bacterias benéficas consiste en microencapsular la bacteria usando biopolímeros funcionales (Ribeiro et al., 2014).

La microencapsulación es un proceso mediante el cual ciertas sustancias bioactivas son retenidas dentro de una matriz o sistema pared con el propósito de protegerlos frente a condiciones ambientales deletéreas (Shoji et al., 2013). Una microcápsula consiste de una membrana fuerte, delgada, semipermeable y esférica que rodea un núcleo sólido o líquido con un diámetro que varía de unas micras a $1 \mathrm{~mm}$. Para preparar las microcápsulas existen numerosas técnicas, y se ha sugerido que podrían identificarse más de 200 métodos; siendo la metodología de gelación iónica un proceso desarrollado principalmente para inmovilizar células bacterianas, además no requiere el uso de elevadas temperaturas ni de solventes orgánicos (Patil et al., 2010). Dentro de las matrices más empleadas para la microencapsulación se encuentra el alginato y la goma gelana (Rosas et al., 2013). Los alginatos (AS) son copolimeros lineales aniónicos compuestos de $\beta$ - D ácido manurónico (M), y $\alpha$-L ácido gulurónico $(G)$ unidos por enlace $\beta$ 1-4 y es estructurado por bloques que pueden ser homopoliméricos ( $M \circ G$ ) o heteropoliméricos (MG) (Lee y Mooney, 2012). Una de las aplicaciones más importantes de los alginatos en biotecnología, es la habilidad que tienen para formar geles estables a través de la interacción de dos cadenas adyacentes $(\mathrm{G})$ con el Ca++ formando zonas de unión que estabilizan la estructura del gel (Fabich et al., 2012), esta estructura generalmente puede ser utilizada como microcápsula. La goma gelana es un heteropolisacarido lineal aniónico compuesto de unidades de repetición de un tetrasacárido (1-3 $\beta$ D glucosa, 1,4 $\beta$ D ácido glucurónico, 1,4 $\beta$ D glucosa, $\alpha-L$ ramnosa) y al igual que los alginatos, la goma gelana también puede formar geles (González et al., 2015). La gelana nativa es conocida como gelana de alto acilo (GAA) debido a que posee un grupo acetato (C6) y un grupo glicerato (C2) en su residuo de glucosa-A. Esta gelana se caracteriza por formar geles blandos y elásticos que pueden ser utilizados en mezclas poliméricas en procesos de gelación iónica con el fin de aumentar la eficiencia de microencapsulación de bacterias probióticas.

Se han llevado a cabo estudios de viabilidad de bifidobacterias microencapsuladas por secado por aspersión sometidas a condiciones gástricas simuladas (Fritzen et al., 2013), de Lactobacillus reuteri microencapsulado utilizando una novedosa tecnología de vibración y bajo condiciones gastrointestinales (De Prisco et al., 2015), pero son pocos los trabajos que se han desarrollado sobre la viabilidad de Lactobacillus delbrueckii microencapsulado en un sistema binario compuesto de gelana de alto acilo y alginato bajo la influencia de jugos gástricos simulados. Actualmente, la microencapsulación de células bacterianas ha ganado atención, ya que algunos autores han logrado incrementar la viabilidad de bacterias probióticas en productos ácidos como el yogurt (Ziar et al., 2012; González et al., 2014). Por tanto, el objetivo del presente estudio es evaluar la viabilidad de Lactobacillus delbrueckii microencapsulado sometido a jugos gástricos simulados utilizando como material pared un sistema binario compuesto por alginato y goma gelana de alto acilo.

\section{MATERIALES Y MÉTODOS}

\section{Materiales}

Lactobacillus delbrueckii LBL 12 fue sumistrado por LB lactis laboratorios (Plovdiv-Bulgaria) usado como principio activo para las microcápsulas. Los agentes encapsulantes fueron alginato (AS) y goma gelana de alto acilo (GAA) suministrados por Modernist pantry (EE.UU). El carbonato de calcio y el agar MRS fue suministrado por Merck Colombia, la pepsina y el surfactante (Span 80) por Sigma Aldrich (St Louis EE.UU), el agar MRS por Oxoid (UK) y el ácido cítrico por Pancreac (USA).

\section{Microencapsulación}


A partir de las dispersiones de los biopolímeros conteniendo una mezcla de L. delbrueckii en una concentración de $8 \log 10 \mathrm{UFC} / \mathrm{ml}$ se llevó a cabo la microencapsulación. Las microcápsulas fueron preparadas por gelación iónica interna, que consiste en la formación de una emulsión agua destilada/aceite vegetal. La fase acuosa fue preparada mezclando $1 \mathrm{~mL}$ de la suspensión celular (L. delbrueckii) con $99 \mathrm{~mL}$ de la dispersión de AS y GAA en proporciones (75 AS/25 GAA\% p/p) previa esterilización en autoclave a $121^{\circ} \mathrm{C}$ durante $\left.15 \mathrm{~min}\right)$. Luego se adiciona carbonato de calcio como donador de $\mathrm{Ca}^{++}$en una proporción de $30 \mathrm{mM}$ de $\mathrm{Ca}^{++}$. Posteriormente la dispersión conteniendo $L$. delbrueckii. AS, GAA y $\mathrm{CaCO}_{3}$ se adiciona en aceite vegetal de girasol conteniendo un $0.2 \%$ v/v de surfactante (Span 80) a una relación (1:2) bajo agitación constante (400 rpm), posterior a los 15 minutos, se incorpora ácido cítrico al sistema hasta alcanzar un pH de 4.2 con el fin obtener las microcápsulas por gelificación de los biopolímeros. Finalmente el aceite es retirado por adsorción y las microcápsulas contenidas en la fase acuosa son centrifugadas a $5000 \mathrm{rpm} / 10 \mathrm{~min}$ dos veces con disolución salina y almacenadas a $4^{\circ} \mathrm{C}$ hasta su uso.

\section{Eficiencia de la microencapsulación}

La suspensión de microcápsulas fue centrifugada a 5000 rpm con el fin de separar las células libres de $L$. delbrueckii. Posteriormente se determinó la concentración bacteriana en el sobrenadante y se calculó la eficiencia de encapsulación (\%EE) con la siguiente formula:

$E E(\%)=(A-B) / A \times 100$

Donde $A$ es la concentración bacteriana total en la suspensión y B es la concentración de la bacteria sin microencapsular encontrada en el sobrenadante.

\section{Exposición a jugos gástricos simulados}

Microcápsulas recién preparadas $(1 \mathrm{~g})$ y $1 \mathrm{~mL}$ de $L$. delbrueckii en estado libre fueron colocados en un tubo de ensayo conteniendo $10 \mathrm{~mL}$ de jugo gástrico simulado $(3 \mathrm{~g} / \mathrm{L}$ pepsina en una solución de cloruro de sodio $0.5 \%$ p/v a pH 2 ajustado con HCl) (Brusch y Záchia, 2011) incubados a $37^{\circ} \mathrm{C}$ durante $240 \mathrm{~min}$.

\section{Determinación de la viabilidad de Lactobacillus delbrueckii}

La viabilidad de L. delbrueckii fue determinada por triplicado empleando el método de recuento en placa utilizando agar MRS como medio de cultivo. Inicialmente se realizaron diluciones seriadas consecutivas de 10 en 10 de la suspensión bacteriana y posteriormente se adicionó $1 \mathrm{~mL}$ de la dilución respectiva en las cajas de petri estériles para finalmente incorporar $15 \mathrm{~mL}$ aproximadamente de agar MRS. Una vez gelifica el agar se llevan las cajas de petri a incubación a $35^{\circ} \mathrm{C}$ durante 48 horas aproximadamente.

\section{Análisis estadístico}

Los experimentos fueron realizados por triplicado. El programa SPSS versión 17.0 para Windows fue utilizado para llevar a cabo el ANOVA (un factor) y establecer las diferencias estadísticas significativas $(P<0.05)$ entre las diferentes viabilidades obtenidas utilizando la prueba de tukey entre los tiempos de exposición.

\section{RESULTADOS Y DISCUSIÓN}

\section{Eficiencia en la microencapsulación de Lactobacillus delbrueckii}

Las microcápsulas obtenidas en el presente estudio utilizando la mezcla binaria de AS y GAA con un $0.2 \%$ (v/v) de surfactante bajo agitación contaste $(400 \mathrm{rpm})$ presentaron una eficiencia en la microencapsulación de L. delbrueckii del $98.7 \%$, estos porcentajes son similares a los expuestos por Rajam y Anandharamakrishnan, (2015) en la microencapsulación de L. plantarum utilizando la técnica de secado por aspersión y proteína de suero lácteo en combinación con fructooligosacáridos como material pared, estos autores encontraron eficiencias cercanas al $98.63 \%$. Este resultado puede ser consecuencia de las propiedades extensibles de la proteína de suero desnaturalizada, que proporciona ventajas al proceso de microencapsulación, como la formación de una película que recubre los poros donde se encontraba el agua evaporada durante el proceso de secado, aumentando así las eficiencias de microencapsulación. Sin embargo, es interesante mencionar que el secado por aspersión es una técnica que requiere de mayor tecnología para la formación de las microcápsulas en comparación a la gelación iónica, ya que el uso de un equipo de secado por aspersión es necesario.

Los resultados sobre eficiencia de microencapsulación presentados en este estudio son mayores a los publicados previamente por González et al., (2013) quienes obtuvieron eficiencias de $94.8 \%$ cuando 
microencapsularon células de Lactococcus lactis a través de un proceso de gelación iónica interna utilizando mezclas de gelana de alto y bajo acilo a una velocidad de agitación de 400 rpm y con adición de $0.2 \%$ de surfactante. Igualmente De Prisco et al., (2015) reportaron que al utilizar tecnología vibrante en la microencapsulación de Lactobacillus reuteri se obtienen eficiencias de microencapsulación cercanas al 97 \% cuando utilizan alginato y mezclas de quitosan. En la microencapsulación por gelificación iónica, el gel resultante es una matriz porosa, por lo cual principios activos microencapsulados son sensibles a condiciones en el medio (Burey et al., 2008; Mortazavian et al., 2007) además, pueden presentar bajas eficiencias de microencapsulación. Por ende, se utilizan mezclas binarias biopoliméricas con el fin de recubrir los poros y disminuir las posibles fugas aumentando así la eficiencia de la microencapsulación (Chávarri et al., 2010). La alta eficiencia de microencapsulación de células bacterianas cuando se utiliza GAA en un $25 \%$ (p/p) obtenida en el presente estudio, puede ser atribuida al mecanismo de gelificación de la GAA, la cual no requiere de iones de calcio para gelificar, solo necesita de calentamiento para iniciar el entrecruzamiento entre las cadenas de gelana que inicia con la disminución de la temperatura obturando de esta manera los poros o fugas que presentan las microcápsulas.

\section{Viabilidad de Lactobacillus delbrueckii sometido a jugos gástricos simulados}

Los resultados de las cinéticas de pérdida de viabilidad de L. delbrueckii microencapsulado y en estado libre durante 240 min de exposición a jugos gástricos simulados se presentan en la tabla 1. En donde se puede apreciar que el material de cobertura utilizado ejerce un efecto protector sobre viabilidad de las bacterias microencapsuladas, debido a que se presentan diferencias estadísticas significativas $(P<0.05)$ entre los porcentajes de viabilidad de L. delbrueckii microencapsulado y en estado libre después de 30 minutos de exposición a fluido gástrico simulado. Similares resultados fueron publicados por Chávarri et al., (2010) quienes incrementaron la viabilidad de L. gasseri y B. bifidum frente a jugos gástricos simulados durante $2 \mathrm{~h}$ a través de la microencapsulación utilizando como material pared mezclas poliméricas binarias de alginato y quitosan.

L. delbrueckii microencapsulado presenta una disminución promedio de su viabilidad cercana al $7 \%$ cada 30 minutos desde el tiempo cero hasta finalizar el tiempo de exposición (4 h) en fluido gástrico simulado, logrando finalmente una viabilidad del 50 \% que corresponde a 4.0 log10 UFC/mL. Kim et al., (2008) observaron resultados similares. Estos autores reportaron una reducción en el conteo de células viables de $L$. acidophilus ATCC 43121 microencapsulado en alginato de sodio sometido a jugos gástricos a pH de 1.2 y 1.5.

Tabla 1: Viabilidad de Lactobacillus delbrueckii sometido a condiciones gástricas simuladas in vitro

\begin{tabular}{|c|c|c|}
\hline Tiempo (minutos) & L. delbrueckii microencapsulado (\%) & L. delbrueckii en estado libre (\%) \\
\hline 0 & $100 \pm 0.12^{\underline{a}}$ & $100 \pm 0.41^{\text {a }}$ \\
\hline 30 & $95 \pm 1.13^{\underline{a}}$ & $75 \pm 1.01^{\mathrm{b}}$ \\
\hline 0 & $87 \pm 1.04^{\underline{a}}$ & $20 \pm 1.23^{\mathrm{b}}$ \\
\hline 90 & $68 \pm 0.97^{\mathrm{a}}$ & $12 \pm 0.38^{\mathrm{b}}$ \\
\hline 120 & $62 \pm 0.28^{\mathrm{a}}$ & $7 \pm 0.74^{\mathrm{b}}$ \\
\hline 150 & $57 \pm 1.03^{\mathrm{a}}$ & $0,5 \pm 0.41^{\mathrm{b}}$ \\
\hline 180 & $57 \pm 0.07^{\mathrm{a}}$ & $0,0 \pm 0.00^{\mathrm{b}}$ \\
\hline 210 & $54 \pm 0.80^{\mathrm{a}}$ & $0,0 \pm 0.00^{\mathrm{b}}$ \\
\hline 240 & $50 \pm 0.44^{\mathrm{a}}$ & $0,0 \pm 0.00^{\mathrm{b}}$ \\
\hline
\end{tabular}

Filas sin ninguna letra en común presentaron diferencias estadísticas a un nivel de confianza del $95 \%$

Con respecto a las bacterias de $L$. delbrueckii en estado libre o sin microencapsular; estas bacterias pierden el $25 \%(2 \log 10 \mathrm{UFC} / \mathrm{mL}$ ) de su viabilidad cuando son expuestas a fluido gástrico simulado durante los primeros 30 minutos y a los 120 minutos de exposición sólo conservan un $7 \%$ su viabilidad que corresponde a $0.56 \log 10$ UFC/mL llegando a perder completamente su viabilidad a los 180 minutos de exposición a jugos gástricos simulados. Es decir, de una concentración inicial de L. delbrueckii en estado libre correspondiente a $8 \log 10 \mathrm{UFC} / \mathrm{mL}$ bajo las condiciones evaluadas en el presente estudio, las bacterias pierden completamente su viabilidad a los 180 minutos de exposición a jugos gástricos simulados, mientras que las bacterias que se encuentran microencapsuladas utilizando AS y GAA como material de pared, conservan en un $57 \%$ su viabilidad que equivale a $4.56 \mathrm{log} U \mathrm{UFC} / \mathrm{mL}$; lo cual supone un efecto protector por parte de las microcápsulas. Similares resultados fueron publicados por Okuro et al., (2013) quienes encontraron que la microencapsulación de Lactobacillus acidophilus junto a prebióticos (inulina y polydextrosa) logra proteger las células de condiciones gástricas simuladas, mientras que las células libres no se detectan después de 210 minutos de exposición. Estos resultados son contrarios a los reportados por otros autores (Hansen et al., 2002; Sultana et al., 2000) quienes encontraron que la microencapsulación no protege efectivamente a los 
microorganismos de bajos valores de $\mathrm{pH}$, esto podría ser una consecuencia de las fugas o poros presentes en las microcápsulas. No obstante, otros estudios encontraron mejor sobrevivencia para L. acidophilus microencapsulado en comparación con células libres cuando son expuestas a soluciones gástricas e intestinales (Chandramouli et al., 2004; Kim et al., 2008; Sabikhi et al., 2010).

Los resultados obtenidos en este estudio demuestran el efecto protector ejercido por la microencapsulación, sugiriendo una disminución en el diámetro de los poros de las microcápsulas, previniendo así la interacción de las células con el jugo gástrico simulado (Mokarram, et al., 2009). Esto implica la potencial aplicación de la microencapsulación con estos materiales de cobertura (AS y GAA) para la preparación de dietas probióticas a base de lactobacilos para reforzar la viabilidad de bacterias benéficas durante su paso a través del estómago, y facilitar la colonización del intestino; por tal motivo la microencapsulación podría ser una alternativa viable en la producción de sistemas alimentarios funcionales.

\section{CONCLUSIONES}

La microencapsulación utilizando mezclas binarias en proporciones de 25 AS/ 75 GAA como material pared logra conservar en un $50 \%$ la viabilidad L. delbrueckii cuando es sometido a jugos gástricos simulados durante 240 minutos. Es posible que la GAA recubra los poros presentes en las microcápsulas aumentando la eficiencia en la microencapsulación e impidiendo la interacción entre el microorganismo y los jugos gástricos simulados. Esto implica la potencial aplicación de la microencapsulación con estos materiales de cobertura (AS y GAA) para la preparación de dietas probióticas a base de lactobacilos para reforzar la viabilidad de bacterias benéficas durante su paso a través del estómago. Sin embargo, se necesitan más estudios sobre la viabilidad de $L$. delbrueckii microencapsulado incorporado en sistemas alimentarios así como estudios de liberación controlada, con el fin de mejorar la aplicación de probióticos en el desarrollo de alimentos funcionales.

\section{REFERENCIAS}

Brusch, G. y M. Záchia, Effect of microencapsulation on survival of Lactobacillus plantarum in simulated gastrointestinal conditions, refrigeration, and yogurt, J Food Eng, 103, 123-128 (2011)

Burey, P. y otros tres autores, Hydrocoloid gel particles: Formation, characterization, and application, Cri Rev Food Sci Nut, 48, 361-377 (2008)

Chandramouli, V. y otros tres autores, An improved method of microencapsulation to protect Lactobacillus spp. in simulated gastric conditions, J Microbiol Met, 56, 27-35 (2004)

Chávarri, M. y otros cinco autores, Microencapsulation of a probiotic and prebiotic in alginate-chitosan capsules improves survival in simulated gastro-intestinal conditions, Int J Food Microbiol, 142, 185-189 (2010)

De Prisco, A. y otros tres autores, Microencapsulation by vibrating technology of the probiotic strain Lactobacillus reuteri DSM 17938 to enhance its survival in foods and in gastrointestinal environment, LWT Food Sci Technol, 61, 452-462 (2015)

Fabich, H. y otros siete autores, Microbial and algal alginate gelation characterized by magnetic resonance, J Biotechnol, 161(3), 320-327 (2012)

Fritzen, C. y otros cuatro autores, Effect of microencapsulation on survival of Bifidobacterium BB-12 exposed to simulated gastrointestinal conditions and heat treatments, LWT - Food Sci Technol, 50, 39-44 (2013)

González, R. y otros dos autores, Obtaining size-controlled microcapsules by ionic gelation with high and low acyl gellans containing Lactococcus lactis, Rev Col Biotecnol, 15(2), 70-80 (2013)

González, R. y otros dos autores, Efecto de la Microencapsulación sobre las Propiedades Reológicas y Fisicoquímicas del Yogurt Blando, Infor Tecnol, 25(6), 45-56 (2014)

González, R. y otros dos autores, Caracterización Viscoelástica de Biopelículas Obtenidas a Base de Mezclas Binarias, Inf. Tecnol, 26(3), en prensa (2015)

Hansen, T. y otros tres autores, Survival of $\mathrm{Ca}$ alginate microencapsulated Bifidobacterium spp, in milk and simulated gastrointestinal conditions, Food Microbiol, 19, 35-45 (2002)

Kim, S. y otros cinco autores, Effect of microencapsulation on viability and other characteristics in Lactobacillus acidophilus ATCC 43121, LWT- Food Sci Technol, 41, 493-500 (2008) 
Lee, K. y D. Mooney, Alginate: Properties and biomedical applications, Progr Pol Sci, 37(1), 106-126 (2012)

Mohammadi, R. y otros tres autores, Probiotic ice cream: Viability of probiotic bacteria and sensory properties, Ann Microbiol, 61, 411-424 (2011)

Mokarram, R. y otros tres autores, The influence of multi stage alginate coating on survivability of potential probiotic bacteria in simulated gastric and intestinal juice, Food Res Int, 42, 1040-1045 (2009)

Mortazavian, A. y otros tres autores, Principles and methods of microencapsulation of probiotic microorganisms, Ir J Biotechnol, 5, 1-18 (2007)

Okuro, $\mathrm{P}$ y otros cinco autores, Co-encapsulation of Lactobacillus acidophilus with inulin or polydextrose in solid lipid microparticles provides protection and improves stability, Food Res Int, 53, 96-103 (2013)

Patil, J. y otros tres autores, lonotropic gelation and polyelectrolyte complexation: The novel techniques to design hydrogel particulate sustained, modulated drug delivery system: A review. Dig J Nanomat Biost, 5, 241-248 (2010)

Rajam, R. y C. Anandharamakrishnan, Microencapsulation of Lactobacillus plantarum (MTCC 5422) with fructooligosaccharide as wall material by spray drying, LWT - Food Sci Technol, 60 773-780 (2015)

Rosas, W. y otros dos autores, Microencapsulation of Lactobacillus helveticus and Lactobacillus delbrueckii using alginate and gellan gum, Carb Poly, 98 1011-1017 (2013)

Ribeiro, M. y otros cinco autores, Effect of microencapsulation of Lactobacillus acidophilus LA-5 on physicochemical, sensory and microbiological characteristics of stirred probiotic yoghurt, Food Res Int, 66, 424-431 (2014)

Sabikhi, L. y otros tres autores, Resistance of microencapsulated Lactobacillus acidophilus LA1 to processing treatments and simulated gut conditions, Food Biopr Technol, 3, 586-593 (2010)

Shoji, A. y otros cinco autores, Viability of $L$. acidophilus microcapsules and their application to buffalo milk yoghurt, Food bioprod proces, 91, 83-88 (2013)

Sultana, K. y otros seis autores, Encapsulation of probiotic bacteria with alginate-starch and evaluation of survival in simulated gastrointestinal conditions and in yogurt, Int J Food Microbiol, 62, 47-55 (2000)

Tripathi, M. y K. Giri, Probiotic functional foods: Survival of probiotics during processing and storage, $J$ Funct Foods, 9, 225-241 (2014)

Zhu, Y. y otros tres autores, Gut microbiota and probiotics in colon tumorigenesis, Can lett, 309, 119-127 (2011)

Ziar, H. y otros dos autores, Calcium alginate-resistant starch mixed gel improved the survival of Bifidobacterium animalis subsp. lactis Bb12 and Lactobacillus rhamnosus LBRE-LSAS in yogurt and simulated gastrointestinal conditions, Int J Food Sci Technol, 47, 1421-1429 (2012) 